\title{
Constraining slepton and chargino through compressed top squark search
}

\author{
Partha Konar, ${ }^{a}$ Tanmoy Mondal ${ }^{b}$ and Abhaya Kumar Swain ${ }^{a, c}$ \\ ${ }^{a}$ Theoretical Physics Group, Physical Research Laboratory, \\ Ahmedabad, 380 009, India \\ ${ }^{b}$ Regional Centre for Accelerator-based Particle Physics, Harish-Chandra Research Institute, HBNI, \\ Chhatnag Road, Jhunsi, Allahabad, 211 019, India \\ ${ }^{c}$ Department of Theoretical Physics, Indian Association for the Cultivation of Science, \\ 2A \&3 2B Raja S.C. Mullick Road, Kolkata, 700 032, India
}

E-mail: konar@prl.res.in, tanmoymondal@hri.res.in, abhaya@prl.res.in

ABSTRACT: We examine the compressed mass spectrum with sub-TeV top squark $(\tilde{t})$ as lightest colored (s)particle in natural supersymmetry (SUSY). Such spectra are searched along with an additional hard jet, not only to boost the soft decay particles, also to yield enough missing transverse momentum. Several interesting kinematic variables are proposed to improve the probe performance for this difficult region, where we concentrate on relatively clean dileptonic channel of the top squark decaying into lightest neutralino $\left(\chi_{1}^{0}\right)$, which is also the lightest supersymmetric particle. In this work, we investigate the merit of these kinematic variables, sensitive to compressed mass region extending the search by introducing additional states, chargino and slepton (sneutrino) having masses in between the $\tilde{t}$ and $\chi_{1}^{0}$. Enhanced production and lack of branching suppression are capable of providing a strong limit on chargino and slepton/sneutrino mass along with top squark mass. We perform a detailed collider analysis using simplified SUSY spectrum and found that with the present LHC data $M_{\tilde{t}}$ can be excluded up to $710 \mathrm{GeV}$ right away for $M_{\chi_{1}^{0}}$ of $640 \mathrm{GeV}$ for a particular mass gap between different states.

KEYWORDS: Supersymmetry Phenomenology

ARXIV EPRINT: 1710.08664 


\section{Contents}

1 Introduction 1

2 Model and framework $\quad 4$

$\begin{array}{lll}3 & \text { Analysis setup and simulation } & 7\end{array}$

4 Result and discussions $\quad 11$

$\begin{array}{llr}5 & \text { Summary and conclusion } & 17\end{array}$

$\begin{array}{ll}\text { A Benchmark points } & 18\end{array}$

\section{Introduction}

The Standard model (SM) of particle physics has been successfully validated in numerous experimental observations with great precision. The latest being the discovery of the SM Higgs boson by CMS [1] and ATLAS [2] at the Large Hadron Collider (LHC). Another important objective of a high energy and high luminosity collider like LHC is to search for new physics and till now LHC has not provided any clinching evidence of new physics. With a strong perception that the SM is a low energy effective theory, lots of effort is poured into constructing models which not only explain experimental observations like dark matter, neutrino oscillation etc, also accommodate some theoretical prejudices like naturalness. Supersymmetry (SUSY) undoubtedly is one of the most explored model in this regard. In a R-parity conserving (RPC) SUSY, the lightest SUSY particle (LSP) is absolutely stable and is a natural DM candidate. In the RPC scenario typical SUSY particles are expected to produce in pair at collider experiment. After cascade decay depending upon possible interactions as well as mass hierarchy, result into a pair of LSPs which are undetectable at the detector. Consequently, SUSY signatures are mostly associated with production of large missing transverse momenta.

The non-observation of any signature of new physics at the LHC effectively constrains a big chunk of SUSY parameter space. Exclusion limits are rather prominent in case of conventional colored particles like squarks and gluino. Currently, light top squark has been excluded up to $1 \mathrm{TeV}$ for the neutralino mass below $160 \mathrm{GeV}[3,4]$. But in order to stabilize the Higgs boson mass from large quantum correction, at least one of the top quark partner (top squark) should be less than one $\mathrm{TeV}$ and expected to show up in the current run of the LHC. Incidentally, these strong constraints from the LHC on top squark weaken considerably if one considers a compressed mass spectrum having narrow mass gap between different sparticle states. One can analyse the situation with full supersymmetry spectrum squeezed enough satisfying available constraints from Higgs measurement and 
dark matter $[5,6]$. However, conventional searches are done considering only simplified two level system where the top squark, the next to lightest supersymmetric particle (NLSP) has mass difference in different degree with the LSP. This region of parameter space is extremely difficult to search at the LHC because of the indistinct kinematics from the SM backgrounds which makes it very challenging to disentangle them. A brief review of the search regions and different strategies pursued in the past is discussed below. The top squark search regions are categorized depending upon the decay kinematics decided by the mass difference between the top squark and LSP. There are mainly two search regions as follows:

Uncompressed top squark. In this region, the top squark $(\tilde{t})$ is heavier compared to the lightest neutralino $\left(\chi_{1}^{0}\right)$ and top mass combined, $M_{\tilde{t}}>M_{t}+M_{\chi_{1}^{0}}$ which allows the two body decay, $\tilde{t} \rightarrow t \chi_{1}^{0}$ or $\tilde{t} \rightarrow b \chi_{1}^{+}$[7] depending on the model. The dominant backgrounds come from top pair, associated top and weak boson pair production. These channels are probed widely by employing numerous kinematical variables, for example, the transverse mass variable, $M_{T 2}[8-12]$, the partonic Mandelstam variable, $\sqrt{\hat{s}_{\min }}[13]$ and its variants $[14,15]$, scalar sum of the transverse momentum of all the reconstructed objects, $H_{T}$ [16], missing transverse momenta (MET), $\mathbb{E}_{T}$, effective mass $M_{\text {eff }}$ [17], invariant mass of various visible particles [17, 18], Razor variable [19-21], $\alpha$ variable [22] and its variant $\alpha_{T}$ [23] etc. Since the top quark in the final state can be very energetic primarily because of the large mass gap between top squark and neutralino, the top quark is boosted and as a result its decay products can be collimated. Jet substructure techniques and large radius fat jet methods were also studied [7].

Compressed top squark. This is the region of parameter space in which the top squark and neutralino mass difference is less than or equal to top quark mass, $M_{\tilde{t}} \lesssim M_{t}+M_{\chi_{1}^{0}}$. Since the mass difference between the top squark and the neutralino is small, the visible decay products produced through off-shell decay are very soft and often fail the selection criteria. Therefore, the principal search channel at the LHC is missing transverse momenta along with a hard jet (ISR) [24-29]. If the top squark has considerable lifetime, it can form stoponium bound state which eventually decays to SM particles. Searching for the stoponium [30-32] is shown to be sensitive to the compressed region of top squark. Compressed top squark is also searched for the case in which it is produced in association with a $Z$ boson or a Higgs from the decay of the heavier top squark assuming that is accessible at the collider [33-36]. Compressed top squarks are also explored along with intermediate sleptons and charginos leading to multi-lepton final state [37, 38].

This compressed region can further be classified into two separate categories depending on the mass difference between the top squark and neutralino. If the mass gap is greater than or equal to $W$ boson and $b$ quark mass combined, $M_{\tilde{t}}-M_{\chi_{1}^{0}}>M_{W}+M_{b}$, then the top squark decays through three body off-shell top decay with on-shell $W$ boson. If the mass gap is even smaller than $W$ boson mass, $M_{\tilde{t}}-M_{\chi_{1}^{0}}<M_{W}$, the top squark decays via four body decay where $W$ is also off-shell, $\tilde{t} \rightarrow b f f^{\prime} \chi_{1}^{0}$ or through loop induced flavor violating two body decay, $\tilde{t} \rightarrow c \chi_{1}^{0}$ [39-41]. Since the charm tagging is not efficient for the flavor violating two body decay, both the CMS and ATLAS rely on the monojet plus 
missing energy search [42-45]. Corresponding limit on top squark mass is $550 \mathrm{GeV}$ using $13 \mathrm{TeV} 36 \mathrm{fb}^{-1}$ data as reported by the CMS [46]. Similarly, in the leptonic decay channel in the region of the four body decay of the top squark, $\tilde{t} \rightarrow b \ell \nu_{\ell} \chi_{1}^{0}$, the CMS and ATLAS collaborations have performed several analyses which includes single lepton and dilepton search in addition to moderate missing transverse momentum [47-50]. The current limit for the single lepton search [51] on the top squark mass is about $500 \mathrm{GeV}$ with $95 \%$ CLs for a mass difference of $30 \mathrm{GeV}$ between the top squark and the LSP. For the dilepton search the limit is $400 \mathrm{GeV}$ with the mass difference $40 \mathrm{GeV}$ [52]. All these limits are calculated with integrated luminosity of $36 \mathrm{fb}^{-1}$.

Recently the variables $R_{M}$ [53] was proposed which is well suited to the compressed region where $M_{\tilde{t}} \simeq M_{t}+M_{\chi_{1}^{0}}$. It exploits the kinematics of the compressed region to explore the hadronic decay of pair of top squarks which recoil against a hard ISR jet. $R_{M}$ is defined as the ratio between missing transverse energy and transverse momentum of the hard ISR jet, $\mathbb{E}_{T} / P_{T}^{\mathrm{ISR}}$. In a compressed region, top squark and the neutralino moves in equal velocity [54] and thus the variable essentially peaks at the ratio between the neutralino and top squark mass. Background from the top pair production does not follow this kinematics and falls exponentially from zero with respect to $R_{M}$. Subsequently, this idea is generalized to accommodate either semi-leptonic or di-leptonic decays of the top squark $[38,55]$. In general, the presence of additional source of missing energy due to presence of neutrinos deteriorate the behavior of the $R_{M}$ leading to decrease in its efficiency. For the semileptonic decay, the neutrino contribution can be subtracted using the available kinematic constraints in each event. Whereas di-leptonic decay channel remains under constraint and thus providing a range of values, but its extremum values found to be useful [38]. Lately, two new variables $R_{b E}$ and $R_{\ell E}$ [56] were proposed for the compressed region of top squark decaying dileptonically. The search strategy using these observables proved to be quite useful in giving a complimentary limit to experimental bounds. Although they were advocated for the four body decay of top squark, these variables are not limited to a particular decay channel as well as a region of parameter space, details about these variables are discussed in the section below. In spite of all these efforts, the current limit at the LHC for this region is still weak especially for the leptonic decay channel of the top squark.

In this paper, we investigate the dilepton channel of the compressed top squark. While this signature is relatively clean and reliable in presence of light leptons (electron, muon), small leptonic branching ratio in this channel constraints weakly compared to the hadronic processes. Hence, the limits coming from typical search with a simplified model where top squark as NLSP is still quite low despite different interesting kinematic variable. Strikingly different result is extracted if one considers two new states chargino and the slepton (sneutrino) in between the top squark and lightest neutralino LSP still within the compressed region. In fact, additional two states make the mass hierarchy even more squeezed. Recently proposed new variables, $R_{b E}$ and $R_{\ell E}$ are found to be extremely effective in this highly compressed scenario. With these observables, the top squark search limit can be significantly improved even for the current LHC data. In addition, these limits can also be translated to constrain the chargino and slepton (sneutrino) masses and we got bounds where there are no or extremely weak experimental limit exist in the parameter space. 


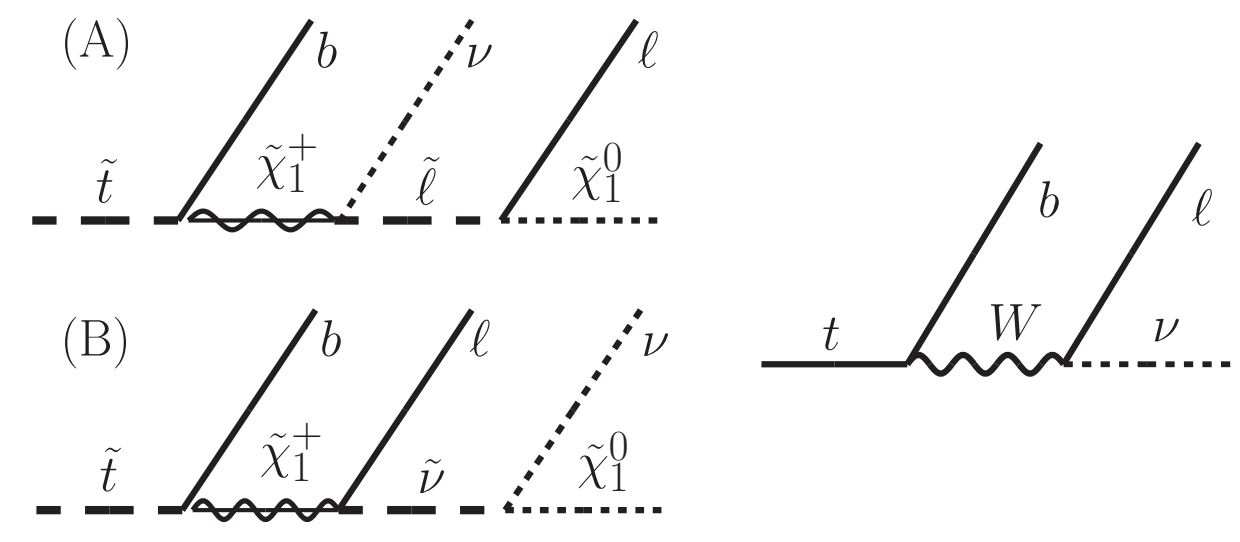

Figure 1. Left panel depicts decay toplogy of the top squark which decays into dileptonic final states via chargino and slepton and sneutrino mediator. For simplicity we show decay of one of the pair produced top squark. In right panel we show decay of the dominant background process $t \bar{t}$.

The rest of the paper is organized in following way. In section 2 we discuss the phenomenological model and the basis for different benchmark points we consider for present analysis. Motivating from distinct signal and background topology suitable kinematic variables are also constructed. Numerical set up and simulation for both the signal and background processes are described in section 3. We also present the effectiveness of different kinematic variables considering representative benchmark scenarios. In section 4 , we present our main results in terms of statistical significance for all the benchmark points we consider. Experimental reach in terms of different masses and mass gaps are demonstrated. Finally, in section 5 we conclude with final points.

\section{Model and framework}

In this analysis, we consider a scenario where the top squark decays predominantly through the dileptonic decay mode. This can be realized, for a SUSY spectrum, if the first two generations of sleptons/sneutrino $(\tilde{\ell} / \tilde{\nu})$ and chargino $\left(\chi_{1}^{ \pm}\right)$lie between the lightest colored (s)particle (LCP), $\tilde{t}$ and the LSP $\left(\chi_{1}^{0}\right)$. Although there are various ways to realize the simplified model, one simple possibility can be a pMSSM scenario where the lightest neutralino is mostly bino type and the lightest chargino is wino type. In this case correct relic abundance can be achieved via bino-slepton coannihilation if the mass gap between neutralino and slepton is small $(<15 \mathrm{GeV})$. For higher mass gap region, one can use the A-funnel region with $m_{A} \sim 2 m_{\chi_{1}^{0}}$ to achieve correct relic density. The Higgs mass constraint can also be satisfied easily by appropriately choosing the trilinear coupling and $\tan \beta$. We have assumed left selectron and left smuons are light and other states are heavier than the lightest stop. We fixed gaugino mass parameter $M_{1}$ and changed $M_{2}$ along with other soft SUSY breaking masses to achieve required mass gaps between $\chi_{1}^{0}$ and $\chi_{1}^{ \pm}$ and lightest stop. The representative diagrams for the dileptonic decay modes of the top squark are shown in the left side of figure 1 . The top squark initially decays to $b$-quark and chargino, and subsequently the chargino decays to either slepton in association with 
a neutrino or a sneutrino along with a lepton. Finally, the slepton (sneutrino) decays to neutralino and a lepton(neutrino). To ensure that the top squark decays predominantly to dileptonic channel we assume that the mass differences respect the suitable mass hierarchy, $M_{\tilde{t}}>M_{\chi_{1}^{+}}>M_{\tilde{\ell} / \tilde{\nu}}>M_{\chi_{1}^{0}}$. Overall mass difference $M_{\tilde{t}}-M_{\chi_{1}^{0}} \equiv \Delta M_{\tilde{t} \chi_{1}^{0}}$ smaller than the top quark mass $M_{t}$ ensures that the uncompressed two body decay mode $\tilde{t} \rightarrow t \chi_{1}^{0}$ is kinematically forbidden. Moreover, individual intermediate mass gaps i.e. $M_{\tilde{t}}-M_{\chi_{1}^{+}} \equiv \Delta M_{\tilde{t}_{\chi_{1}^{+}}}$ and $M_{\chi_{1}^{+}}-M_{\tilde{\ell} / \tilde{\nu}}=M_{\tilde{\ell} / \tilde{\nu}}-M_{\chi_{1}^{0}} \equiv \Delta M_{\chi_{1}^{+} \tilde{\ell}}$ are also small due to the compressed nature of the considered simplified model. We would vary these mass differences in different range and discuss the consequence in subsequent sections. It is evident that the following relation always holds:

$$
\Delta M_{\tilde{t} \chi_{1}^{0}}=\Delta M_{\tilde{t} \chi_{1}^{+}}+2 \times \Delta M_{\chi_{1}^{+} \tilde{\ell}}
$$

where we have assumed that the slepton and sneutrino are mass degenerate and lie exactly in the middle of mass gap between chargino and LSP. These assumptions are made to ensure an enhanced production of signal events and avoid the production of very soft final state particles leading to efficient detection. The branching fraction of wino-like chargino $\chi_{1}^{+}$decaying to slepton or sneutrino depends on the diagonalizing matrices. However, since the second chargino is very heavy, we assume that the $\chi_{1}^{+}$decays democratically to both sleptons and sneutrinos i.e. $\operatorname{BR}\left(\chi_{1}^{+} \rightarrow \ell \tilde{\nu}\right)=\operatorname{BR}\left(\chi_{1}^{+} \rightarrow \nu \tilde{\ell}\right)=50 \%$.

One important feature of the assumed spectrum is that the top squark pair decays to dilepton without any substantial branching ratio suppression, whereas in the conventional compressed SUSY case the top squark pair decays to dilepton via off-shell decay of the $W$ boson which has relatively small branching ratio to leptons $(\sim 20 \%)$. Thus, the particular channel that we consider in this work is capable of delivering strong limit on top squark mass which in turn can constrain chargino and sleptons/sneutrinos mass even within the framework of compressed spectrum. Here we want to emphasize that though the top squark proceeds via sequential two body decay, the phase space available to each decay is highly compressed because of the two additional resonant states present in between the top squark and LSP. In addition to the cross-section enhancement, the kinematic variables, which are suitably constructed for such scenario, are also very effective in this region because of the high level of compression in the spectrum. Now we describe these variables before utilizing them in our analysis in the next section.

We considered two new and simple kinematic variables which are very effective in the compressed region in discriminating signal and background. These variables were earlier proposed in our previous work [56] where we have studied the four body decay of the top squark in the dileptonic channel. These variables are defined as the ratio between vector sum of all $b$ jet (lepton) transverse momentum and missing transverse momenta as follows,

$$
R_{b E}=\frac{\left|\sum \vec{P}_{T}^{b_{i}}\right|}{\mathbb{E}_{T}}, \quad R_{\ell E}=\frac{\left|\sum \vec{P}_{T}^{\ell_{i}}\right|}{\mathbb{E}_{T}} .
$$

These variables are proved to be useful to distinguish the compressed top squark from the large $t \bar{t}$ background. Now allowing electroweak states within such a narrow mass gap for a successive two body decay, we argue that these ratio variables can still offer as good as, if not better, discrimination compare to the conventional scenario. 
In our present decay chain as shown in figure 1, when the top squark decays to $b \chi_{1}^{ \pm}$if the mass difference is such that $M_{\tilde{t}}=M_{b}+M_{\chi_{1}^{ \pm}}$then both the b-quark and the chargino have the same velocity vector at the equal-velocity limit. Heavier top squarks dominantly produced at the threshold and there by originate soft decay products if the mass difference are small. However, if the top squark recoils against a high $p_{T}$ ISR then the decay particles will have moderate transverse energy and owing to the equal velocity approximation we can write

$$
p_{T}(b)=\frac{M_{b}}{M_{\tilde{t}}} p_{T}(\tilde{t}), \quad p_{T}\left(\chi_{1}^{ \pm}\right)=\frac{M_{\chi_{1}^{ \pm}}}{M_{\tilde{t}}} p_{T}(\tilde{t}) .
$$

Furthermore if we assume the approximation holds for the subsequent decay of chargino and slepton then the following relation holds:

$$
p_{T}(\tilde{\ell})=\frac{M_{\tilde{\ell}}}{M_{\chi_{1}^{ \pm}}} p_{T}\left(\chi_{1}^{ \pm}\right) \quad \text { and } \quad p_{T}\left(\chi_{1}^{0}\right)=\frac{M_{\chi_{1}^{0}}}{M_{\tilde{\ell}}} p_{T}(\tilde{\ell}) .
$$

Using the equation (2.3) and (2.4) we have,

$$
\begin{aligned}
\sum p_{T}\left(b_{i}\right) & =\frac{M_{b}}{M_{\tilde{t}}}\left(p_{T}(\tilde{t})+p_{T}\left(\tilde{t}^{*}\right)\right)=\frac{M_{b}}{M_{\tilde{t}}} \cdot \frac{M_{\tilde{t}}}{M_{\chi_{1}^{ \pm}}} \sum p_{T}\left(\chi_{1}^{ \pm}\right) \\
& =\frac{M_{b}}{M_{\chi_{1}^{ \pm}}} \cdot \frac{M_{\chi_{1}^{ \pm}}}{M_{\tilde{\ell}}} \sum p_{T}(\tilde{\ell})=\frac{M_{b}}{M_{\tilde{\ell}}} \cdot \frac{M_{\tilde{\ell}}}{M_{\chi_{1}^{0}}} \sum p_{T}\left(\chi_{1}^{0}\right),
\end{aligned}
$$

where $\sum p_{T}\left(\chi_{1}^{0}\right)$ is the dominant component of the missing energy $\mathbb{E}_{T}$. The same exercise can be done for $\sum p_{T}\left(\ell_{i}\right)$ which results into $\frac{M_{\ell}}{M_{\chi_{1}^{0}}} \sum p_{T}\left(\chi_{1}^{0}\right)$. Above arguments should suffice to demonstrate that the consecutive application of equal-velocity approximations ultimately cancel the mass dependence of intermediate states, so that the final algebraic relation remains as proposed in [56]. Evidently, these variables are well suited to study the signature considered here.

In the present study the phase space available for the $b$-jet and the lepton is determined by the mass gaps $\Delta M_{\tilde{t} \chi_{1}^{+}}$and $\Delta M_{\chi_{1}^{+} \tilde{\ell}}$ respectively. Although the exact mass relation for equal velocity approximation does not hold, since the phase space is completely determined by the mass gaps, the information can be used judiciously for the demarcation of signal and background events. For the background events, the b-jets are originated from the top decay and the leptons are stemmed from the decay of an on-shell $W$ boson. We demonstrate that although the variables $R_{b E}$ and $R_{\ell E}$ were initially proposed for the search of the four body decay region of the top squark, these variables are versatile enough to be used in different search channels like the present scenario at the LHC with the compressed spectrum.

In addition to the $R_{b E}$ and $R_{\ell E}$, the invariant mass variable between $b$ and $\ell$ system also encodes the mass differences between the mediators. When the top squark decays via sleptons as shown in figure $1(\mathrm{~A})$, then the endpoint of the $b \ell$ system invariant mass is given by $[57]$

$$
\left(M_{b \ell}^{\max }\right)_{\tilde{\ell}}^{2}=\frac{\left(M_{\tilde{t}}^{2}-M_{\chi_{1}^{+}}^{2}\right)\left(M_{\tilde{\ell}}^{2}-M_{\chi_{1}^{0}}^{2}\right)}{M_{\chi_{1}^{+}}^{2}} .
$$


On the other hand if there is sneutrino in the mediator, as in figure 1(B), then the invariant mass distribution of bl system have an endpoint at [57]

$$
\left(M_{b \ell}^{\max }\right)_{\tilde{\nu}}^{2}=\frac{\left(M_{\tilde{t}}^{2}-M_{\chi_{1}^{+}}^{2}\right)\left(M_{\chi_{1}^{+}}^{2}-M_{\tilde{\nu}}^{2}\right)}{M_{\chi_{1}^{+}}^{2}} .
$$

Hence, depending on the mediator the endpoint will change for a particular $b \ell$ combination. For a given spectrum with degenerate slepton and sneutrino masses, it is evident from above equations that the sneutrino mediated quantity is always larger than the other, i.e. $\left(M_{b \ell}^{\max }\right)_{\tilde{\nu}}>\left(M_{b \ell}^{\max }\right)_{\tilde{\ell}}$. In a compressed scenario like we have considered here, this difference is typically below a $\mathrm{GeV}$, and we estimate the theoretical endpoint $M_{b \ell}^{\max }$ as a event selection parameter by considering the largest of these two. It is worth noting that the variable $R_{\ell E}$ and $M_{b \ell}$ are quite sensitive to the mass gap between the intermediate resonances. Moreover, $R_{\ell E}$ is sensitive only to $\Delta M_{\chi_{1}^{+} \tilde{\ell}}$ while $M_{b \ell}$ depends on both $\Delta M_{\tilde{t}_{1}^{+}}$ and $\Delta M_{\chi_{1}^{+} \tilde{\ell}}$.

The background for the dilepton channel with at least one b-tagged jets comes predominantly from $t \bar{t}$ with sub-leading contribution comes from $t W$ channel. The $t \bar{t}$ background decay chain is shown in right panel of figure 1 . For the background process the $b \ell$ invariant mass has a endpoint at $\sqrt{M_{t}^{2}-M_{W}^{2}} \simeq 153 \mathrm{GeV}$ which is way larger than the $M_{b \ell}^{\max }$ for signal events in the compressed spectrum. In the next section we show how these variables can be used to minimize the backgrounds.

\section{Analysis setup and simulation}

We consider the pair production of top squark associated with a hard (ISR) jet against which the top squark pair recoils. We are looking into the following signal process,

$$
P P \rightarrow 2 \ell+\geq 1 \text { b-tagged-jet }+\geq 1 \text { high-PT jet (ISR) }+\mathbb{E}_{T} .
$$

To simulate the signal events we assume a simplified model with the mass hierarchy $M_{\tilde{t}}>M_{\chi_{1}^{+}}>M_{\tilde{\ell} / \tilde{\nu}}>M_{\chi_{1}^{0}}$ and two body successive decay to produce above mentioned signature. The signal events as well as the background events are generated using MadGraph5_aMC@NLO [58] with NN23L01 [59] parton distribution function and with the default dynamic renormalisation and factorisation scales [60] in MadGraph5_aMConLO. Pythia8 [61,62] is used for showering and hadronization of the parton level events and subsequently full detector level simulation is done in Delphes3 [63]. All the samples are matched up to one jet using MLM scheme [64, 65]. For the entire analysis we have used the next-to-leading-order (NLO) plus next-to-leading-logarithm (NLL) top squark crosssections given by the LHC SUSY cross-section Working Group [66, 67].

The SM processes which can mimic the signal can come from $t \bar{t}$ and $t W$ processes where the top decays leptonically. We have also simulated these background events using MadGraph5_aMC@NLO followed by Pythia8 and Delphes3 and are matched up to one jets using MLM scheme. The $t \bar{t}$ production cross-section is $\sigma_{t \bar{t}}=815.96 \mathrm{pb}$ as calculated with the 
Top ++2.0 program to next-to-next-to-leading order in perturbative QCD, including softgluon resummation to next-to-next-to-leading-log order (see [68] and references therein), and assuming a top-quark mass $m_{t}=173.2 \mathrm{GeV}$. For the $t W$ channel the NLO+NNLL cross-section is $71.7 \mathrm{pb}$ [69]. It is obvious that the dominant contribution to the background events are coming from the $t \bar{t}$ events due to large production cross-section.

The jets are reconstructed using the anti-kT algorithm [70] with jet radius $R=0.4$. We tagged the highest $p_{T}$ jet as the ISR jet and we require $p_{T}(\mathrm{ISR})>100 \mathrm{GeV}$. A moderately high ISR ensures that the pair produced top squarks recoil against the ISR and produce large missing transverse energy. In order to minimize the QCD background we use the fact that the ISR and MET are in opposite direction for signal events and we apply the cut $\mid \Delta \phi\left(\mathbb{E}_{T}\right.$, ISR $) \mid<0.5$ where $\Delta \phi\left(\mathbb{E}_{T}\right.$, ISR $)=\phi\left(\mathbb{E}_{T}\right)-\phi($ ISR $)-\pi$. We also demand $\left|\phi(j)-\phi\left(\mathbb{E}_{T}\right)\right|>0.2$ for all jets other than the ISR in order to minimize the effect of jet mis-measurement contributing into $\mathbb{E}_{T}$.

For b-jet tagging we use the Combined Secondary Vertex algorithm at the Medium operating point(CSVM) since the tagging algorithm delivers the best efficiency at medium operating point [71]. The b-tagging efficiency depends on the transverse momentum of the jet and parametrized in Delphes3 as,

$$
\mathcal{E}_{\mathrm{b} \text {-tag }}=0.85 \tanh \left[0.0025 p_{T}(j)\right] \frac{25.0}{1+0.063 \times p_{T}(j)},
$$

where $p_{T}(j)$ is the jet transverse momentum and $p_{T}(j) \geq 20 \mathrm{GeV}$. The tagging efficiency is close to $50 \%$ for low $p_{T}$ jets and peaks at $p_{T}(j) \simeq 150 \mathrm{GeV}$ where $\mathcal{E}_{b} \simeq 73 \%$. Mis-tagging efficiency of the light jets is parametrized as

$$
\mathcal{E}_{\text {mis-tag }}=0.01+0.000038 p_{T}(j)
$$

which increases linearly and becomes $1.5 \%$ for $p_{T}(j) \simeq 150 \mathrm{GeV}$. Since we are interested in the compressed spectrum, the b-jets tend to have low $p_{T}$ and hence due to less efficiency it is not economical to tag both the b-jets.

The transverse momentum of the leptons depends on the mass differences $\Delta M_{\chi_{1}^{+} \tilde{\ell}}$ and can be very soft. The CMS collaboration has studied the prospect of soft leptons in compressed top squark search [50] where varying light lepton selection efficiency was used depending on the transverse momentum and pseudorapidity of the leptons [72]. The electron should have a minimum $p_{T}(e)$ of $5 \mathrm{GeV}$, whereas the muons are required to have $p_{T}(\mu)$ at least $3.5 \mathrm{GeV}$. For a realistic simulation we have used the exact lepton identification efficiencies used by the CMS collaboration [72]. The exact values of efficiencies are shown in figure 2 for different $p_{T}-\eta$ bin. As one can see the efficiencies for very soft leptons are small and overall efficiency to tag an electron is smaller than that of muons.

The kinematics of the leptons and b-jet depend strongly on mass gaps $\Delta M_{\chi_{1}^{+} \tilde{\ell}}$ and $\Delta M_{\tilde{t} \chi_{1}^{+}}$. Since the minimum transverse momentum required by a tagged b-jet needs to have $20 \mathrm{GeV}$, the minimum $\Delta M_{\tilde{t}_{1}^{+}}$should be close or greater than the same to allow enough phase space for the $b$ - quark. In similar argument we set the benchmark points such that the $\Delta M_{\chi_{1}^{+} \tilde{\ell}}$, which controls the lepton $p_{T}$, required to have minimum value of $10 \mathrm{GeV}$. Using the present search strategy it is extremely difficult to search compressed 


\begin{tabular}{|c|c|c|c|c|c|c|c|c|c|}
\hline $50-200$ & 0.729 & 0.698 & 0.330 & 0.541 & 0.417 & 0.857 & 0.863 & 0.837 & 0.721 \\
\hline $40-50$ & 0.719 & 0.682 & 0.342 & 0.525 & 0.400 & 0.847 & 0.846 & 0.819 & 0.700 \\
\hline $30-40$ & 0.704 & 0.666 & 0.337 & 0.508 & 0.368 & 0.839 & 0.835 & 0.809 & 0.681 \\
\hline $25-30$ & 0.686 & 0.645 & 0.345 & 0.492 & 0.348 & 0.831 & 0.824 & 0.789 & 0.662 \\
\hline $20-25$ & 0.668 & 0.631 & 0.316 & 0.482 & 0.334 & 0.823 & 0.808 & 0.777 & 0.643 \\
\hline $15-20$ & 0.659 & 0.604 & 0.319 & 0.449 & 0.301 & 0.812 & 0.792 & 0.754 & 0.585 \\
\hline $10-15$ & 0.574 & 0.535 & 0.300 & 0.381 & 0.246 & 0.800 & 0.770 & 0.724 & 0.515 \\
\hline $5-10$ & 0.503 & 0.422 & 0.202 & 0.268 & 0.166 & 0.688 & 0.670 & 0.586 & 0.358 \\
\hline $3.5-5$ & 0 & 0 & 0 & 0 & 0 & 0.688 & 0.670 & 0.586 & 0.358 \\
\hline & $0^{\infty}$ & $0^{\infty} 0^{\alpha}$ & $\lambda^{\alpha^{\prime}}$ & 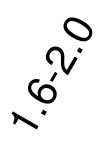 & $\overbrace{}^{0^{2}}$ & $\begin{array}{l}9 \\
0^{0} \\
0^{\circ}\end{array}$ & $0^{9}$ & $\imath^{\hat{v}}$ & $\hat{i}^{\eta^{i}}$ \\
\hline
\end{tabular}

Figure 2. Tagging efficiencies for leptons in different $p_{T}-\eta$ bin [72]. Minimum $p_{T}$ for electron is $5 \mathrm{GeV}$ and for muons it is $3.5 \mathrm{GeV}$. The efficiencies for very soft leptons are small and overall efficiency to tag an electron is less than that of muons.

spectrum below the minimum values of $\Delta M_{i j}$ chosen here and we may need different strategy like search for long lived charged tracks etc. From these minimum values of $\Delta M_{i j}$ it is evident that we can not search for compressed region where overall mass difference $\Delta M_{\tilde{t} \chi_{1}^{0}}$ is less than $40 \mathrm{GeV}$ (see equation (2.1). Moreover, b-jet tagging efficiency is poor for small $P_{T}$ regions which we are actually interested to probe and hence our method can only probe stop-chargino mass gap above $20 \mathrm{GeV}$ (as evident in figure 6). Any further improvements in low $P_{T}$ b-tag can enable us to probe low mass gap region. Secondly, $\mathbb{E}_{T}$ is calculated indirectly from the transverse momentum imbalance of all the isolated objects such as leptons, photons and jets, as well as any unclustered deposits. Accuracy of this measurement is susceptible to underlying events especially the pile up in high luminosity.

The phase space available for the leptons depend on the mass gap $\Delta M_{\chi_{1}^{+} \tilde{\ell}}$ and consequently the $R_{\ell E}$ distribution varies with it. We found that as $\Delta M_{\chi_{1}^{+} \tilde{\ell}}$ increases the $R_{\ell E}$ distribution move towards higher values and upper edge of the distribution follows the following empirical relation:

$$
R_{\ell E}^{\max }=0.0576+0.0047 \frac{\Delta M_{\chi_{1}^{+}} \tilde{\ell}}{\mathrm{GeV}} .
$$

We have used this relation to find $R_{\ell E}^{\max }$ for a particular $\Delta M_{\chi_{1}^{+} \tilde{\ell}}$ in each benchmark point. This criteria is very effective in minimizing the backgrounds as for the SM processes the leptons are originated from the on-shell $W$ boson and tends to have higher values of $R_{\ell E}$. 

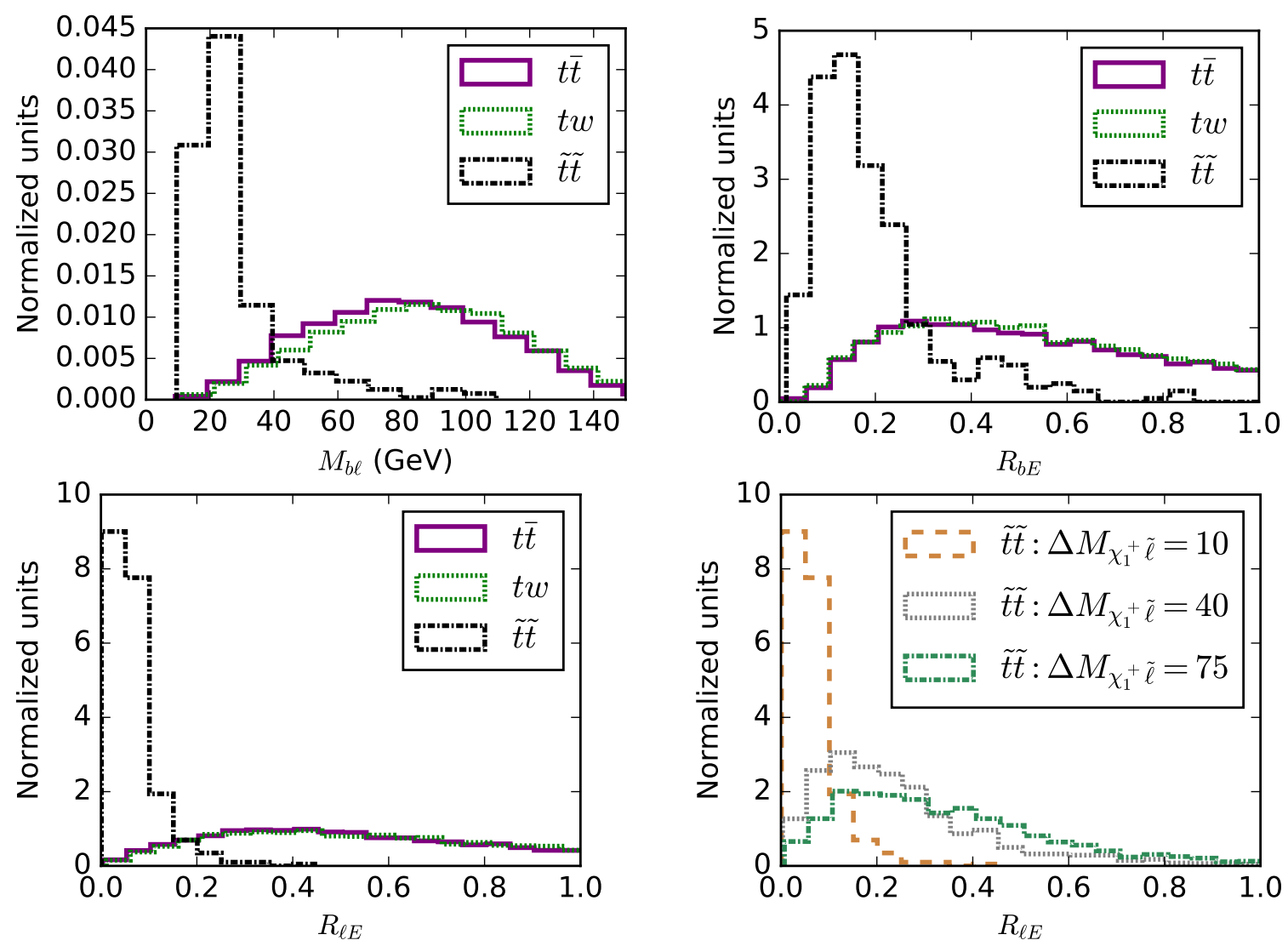

Figure 3. Normalized distributions of different relevant kinematic variables $M_{b \ell}, R_{b E}$ and $R_{\ell E}$ which are used in the present analysis to get improved signal to background ratio. Black (dash-dot) distribution lines in each plot is for signal top squark pair production, along with two dominant background processes. All the plots except the bottom right one are generated for a representative benchmark point with $\Delta M_{\tilde{t} \chi_{1}^{+}}=20 \mathrm{GeV}$ and $\Delta M_{\chi_{1}^{+} \tilde{\ell}}=10 \mathrm{GeV}$. The bottom right plot illustrates the variation on the signal distribution on the mass difference $\Delta M_{\chi_{1}^{+} \tilde{\ell}}$ in $\mathrm{GeV}$. For all the distributions it is easy to observe that the background and signal processes are well separated and the lower the $\Delta M_{i j}$, the bigger the separation between signal and backgrounds.

Similarly, $R_{b E}$ distribution depends on $\Delta M_{\tilde{t} \chi_{1}^{+}}$but the dependency is mild. To keep our presentation simple for the present analysis we fix it to a constant value $R_{b E}^{\max }=0.2$.

In figure 3 we have presented different variables which are used to minimize the background. For this demonstration, all the plots are generated considering $\Delta M_{\tilde{t} \chi_{1}^{+}}=20 \mathrm{GeV}$ and $\Delta M_{\chi_{1}^{+} \tilde{\ell}}=10 \mathrm{GeV}$. While these distributions are independent of the top squark mass, here we took its value as $700 \mathrm{GeV}$. The bottom right plot illustrates the variation on the distribution of $R_{\ell E}$ based on the mass difference $\Delta M_{\chi_{1}^{+} \tilde{\ell}}$. As $\Delta M_{\chi_{1}^{+} \tilde{\ell}}$ increases, the transverse momentum of the leptons also increases leading to a larger value of $R_{\ell E}$. From the distributions it is quite comprehensible that the background and signal processes are well separated and the lower the $\Delta M_{i j}$, the bigger the separation between signal and backgrounds. This leads to better signal to background ratio for lower mass gap regions. However, the lower mass gap region is inherently adverse due to lower signal acceptance factor (since the final 


\begin{tabular}{|c|c|c|c|c|}
\hline Cut & Signal & \multicolumn{2}{|c|}{ Background } & Significance \\
& & $t \bar{t}$ & $t W$ & @300fb $^{-1}$ \\
\hline $\begin{array}{c}\text { Pre-selection+ } \\
2 \ell+\mathrm{ISR}+\geq 1 b\end{array}$ & $371[100 \%]$ & $348680[100 \%]$ & $40090[100 \%]$ & 0.6 \\
\hline$E_{T}>200 \mathrm{GeV}$ & $177[47.8 \%]$ & $17280[4.9 \%]$ & $3464[8.6 \%]$ & 1.2 \\
\hline$\left|\Delta \phi\left(\mathbb{E}_{T}, \mathrm{ISR}\right)\right|<0.5$ & $153[41.3 \%]$ & $12840[3.7 \%]$ & $2364[5.9 \%]$ & 1.2 \\
\hline$M_{b \ell}<50$ & $130[35.2 \%]$ & $2661[0.8 \%]$ & $277[0.7 \%]$ & 2.4 \\
\hline$R_{b E}<0.2$ & $112[30.2 \%]$ & $1415[0.4 \%]$ & $155[0.4 \%]$ & 2.8 \\
\hline$R_{\ell E}<0.152$ & $100[27.0 \%]$ & $325[0.09 \%]$ & $19[0.05 \%]$ & 5.2 \\
\hline
\end{tabular}

Table 1. The effectiveness of different variables to minimize the colossal background is shown in this cutflow table. This representative signal is generated for $\Delta M_{\tilde{t} \chi_{1}^{+}}=30 \mathrm{GeV}$ and $\Delta M_{\chi_{1}^{+} \tilde{\ell}}=20 \mathrm{GeV}$ with the fixed the top squark mass at $725 \mathrm{GeV}$. All the number of events for signal and background are generated with integrated luminosity of $300 \mathrm{fb}^{-1}$. The maximum qualifying values for $M_{b \ell}$ and $R_{\ell E}$ are selected according to the mass gaps as described in the text. $R_{b E}^{\max }$ is kept constant for all benchmark points. As one can see due to narrow mass gap in the mass spectra the variables efficiently retain at least $27 \%$ of the signal events while discarding more than $99.9 \%$ of the background events leading to $5 \sigma$ significance.

state particles are soft) which to some extend normalizes the improvement achieved from the well separated signal and backgrounds. Top left panel of figure 3 represents the invariant mass of $b$-jet and lepton. Since we have tagged only one $b$-jet, there are two possible $b \ell$ combination and we choose the smaller value among those two to calculate the $M_{b \ell}$. For the given $\Delta M_{\tilde{t}_{1}^{+}}, \Delta M_{\chi_{1}^{+} \tilde{\ell}}$ and the top squark mass in the shown example, $M_{b l}^{\max }$ turns out to be around $28 \mathrm{GeV}$ where difference between two equations is in sub-GeV.

\section{Result and discussions}

In previous sections we discussed the model framework and characteristic kinematic distributions which can be utilized to improve the signal to background ratio. To quantify the efficacy of different proposed kinematic variables we consider a representative benchmark point with $\Delta M_{\tilde{t}_{1}^{+}}=30 \mathrm{GeV}$ and $\Delta M_{\chi_{1}^{+} \tilde{\ell}}=20 \mathrm{GeV}$, i.e. total mass gap between top squark and the lightest neutralino is below the $W$ boson mass $M_{W}$. Detailed step by step cut flow is presented in table 1 corresponding to this signal point along with the backgrounds. The events are estimated with an integrated luminosity of $300 \mathrm{fb}^{-1}$ and the signal events are generated with top squark mass $725 \mathrm{GeV}$. As mentioned before, the exact choice of $M_{b \ell}$ selection criteria is decided by the mass gaps as in equations (2.6) and (2.7). Similarly, the maximum allowed value of $R_{\ell E}$ varies according to equation (3.4) primarily depending on $\Delta M_{\chi_{1}^{+} \tilde{\ell}}$. The choice of $R_{b E}$ cut also, in principle, depends on $\Delta M_{\tilde{t} \chi_{1}^{+}}$. For the sake of simplicity, we fix the maximum allowed value of $R_{b E}$ at 0.2 to select our events. With these sequential cuts we have analyze the signal $(S)$ and background events $(B)$, and also present the corresponding statistical significance $(\mathcal{S})$ at each step in the right most column. 
We estimate the $\mathcal{S}$ using the well known expression:

$$
\mathcal{S}=\sqrt{2\left[(S+B) \ln \left(1+\frac{S}{B}\right)-S\right]} .
$$

Since the presence of lightest neutralino in the final state results larger $\mathbb{E}_{T}$ for signal events compared to the background, we have chosen minimum MET of $200 \mathrm{GeV}$ which minimizes the background considerably. However, the dominant background cross-section is three-orders of magnitude higher than the signal cross-section and it is evident from the significance column that only MET cut (which is the usual search strategy for compressed spectrum) is not enough to size the background. As we introduce the new kinematic variables the background events drop rapidly, consequently the significance increases steadily and reaches $5 \sigma$. This cut flow table demonstrates the usefulness of the proposed variables.

Now to explore the full model parameter space in terms of two relevant mass differences $\Delta M_{\tilde{t} \chi_{1}^{+}}$and $\Delta M_{\chi_{1}^{+} \tilde{\ell}}$, we vary both these mass gaps in such a way that the total mass difference between top squark and the lightest neutrino $\Delta M_{\tilde{t}_{1}^{0}}$ does not go beyond the top quark mass. To achieve this we vary the mass difference $\Delta M_{\tilde{t} \chi_{1}^{+}}$in between $20 \mathrm{GeV}$ to $70 \mathrm{GeV}$ with a step of $10 \mathrm{GeV}$ and $\Delta M_{\chi_{1}^{+}} \tilde{\ell}$ is chosen accordingly. The maximum allowed value of the parameters $M_{b \ell}$ and $R_{\ell E}$ changes according the mass gaps. Using the new kinematic variables we calculated the $2 \sigma$ exclusion limit at $13 \mathrm{TeV}$ LHC with integrated luminosity of $36 \mathrm{fb}^{-1}$ and is depicted in the heat plot in figure 4 . The abscissa is showing the mass gap between top squark and chargino $\Delta M_{\tilde{t} \chi_{1}^{+}}$which is responsible for the b-jet phase space and the ordinate is half of the chargino neutralino mass difference $\Delta M_{\chi_{1}^{+} \tilde{\ell}}$ which controls the lepton $p_{T}$ spectrum. The color bar is showing the top squark mass which can be excluded for given mass gaps in both the axes. For readability, we put few contours with fixed top squark mass. The diagonal dotted lines represent a fixed top squark-neutralino mass gap which are indicated with $\Delta M_{\tilde{t} \chi_{1}^{0}}=170,140,100$ and $70 \mathrm{GeV}$. Along these dotted lines all the off-white points are the benchmark points we have chosen to scan the parameter space. Among these benchmark points, two white stars marks are indicated for which we supplied the complete mass spectrum at the appendix table 3 , satisfying all the collider, DM and low energy constraints. All the benchmark points and the corresponding cut values of different variables are tabulated later in table 2 .

It is evident from the figure 4 that the LHC reach is more sensitive to the vertical axis which regulates the leptonic phase space. As we move towards the higher values in vertical axis, the leptons tend to have larger transverse momentum which increase $R_{\ell E}^{\max }$. Since the dominant $t \bar{t}$ background tends to have $R_{\ell E}$ distribution which peaks at a higher value, increase in $R_{\ell E}^{\max }$ results in decreasing the LHC reach. For different values of the horizontal axis the b-tagged jet $p_{T}$ varies its effect in the final significance is mild. However, it is obvious that our proposed variables works well at the lower end of the mass difference which is very difficult region to explore. The white region in the top right corner has not been explored in this work since we adhere to the fact that the top squark neutralino mass difference should not go beyond the so called top-threshold, beyond which the 2-body top squark decay $\tilde{t} \rightarrow t \chi_{1}^{0}$ is important and should be taken into account to achieve a realistic limit on the top squark mass. 


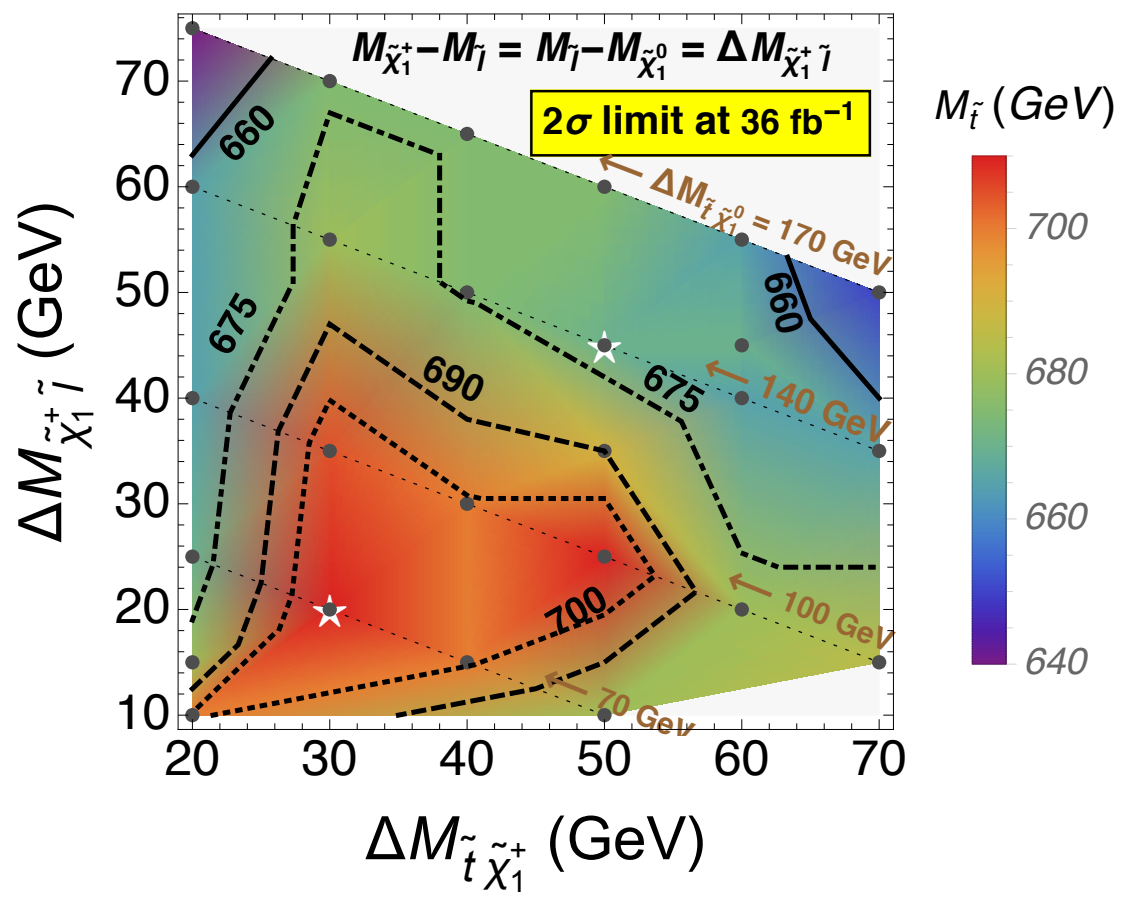

Figure 4. Reach of $13 \mathrm{TeV}$ LHC with $36 \mathrm{fb}^{-1}$ data for different values of different mass gaps between top squark, chargino and the neutralino. The horizontal axis is responsible for the b-jet $p_{T}$, while the vertical axis controls the lepton phase space. The white space in top right corner is not analyzed as in this region the top squark - neutralino mass difference goes beyond the top-corridor. The bottom right white space is numerical artifact due to computational constraints.

To further understand the implication of the present bound, we have plotted in figure 5 the exclusion limits in the $M_{\tilde{t}}-M_{\chi_{1}}^{0}$ plane along with other exclusion limits obtained from different compressed searches. The purple bold line depicts the minimum top squark mass which can be excluded at $2 \sigma$ at $13 \mathrm{TeV}$ LHC with integrated luminosity of $36 \mathrm{fb}^{-1}$ data for a given top squark-neutralino mass gap provided $\Delta M_{\tilde{t} \chi_{1}^{+}}$and $\Delta M_{\chi_{1}^{+} \tilde{\ell}}$ lie within their range of our described benchmark points. ${ }^{1}$ The best exclusion limit on top squark mass is achieved for a particular $\Delta M_{\tilde{t} \chi_{1}^{+}}$and $\Delta M_{\chi_{1}^{+} \tilde{\ell}}$ combination which is the most sensitive to the kinematic variables. This limit is shown by the purple dotted line. For clarification one can follow from the heat map plot in figure 4 . The best limit on top squark mass is $710 \mathrm{GeV}$ and is achieved for $\Delta M_{\tilde{t}_{1}^{+}}=30 \mathrm{GeV}$ and $\Delta M_{\chi_{1}^{+} \tilde{\ell}}=20 \mathrm{GeV}$, whereas the minimum limit is $640 \mathrm{GeV}$ and with $\Delta M_{\tilde{t}_{1}^{+}}=20 \mathrm{GeV}$ and $\Delta M_{\chi_{1}^{+} \tilde{\ell}}=75 \mathrm{GeV}$. They represent two points over these dashed and solid purple curves respectively.

Anything between these two lines can be excluded for various $\Delta M_{\tilde{t} \chi_{1}^{+}}$and $\Delta M_{\chi_{1}^{+} \tilde{\ell}}$ combination and are shown with light purple hue. Similarly orange curves and the light orange color band is presented for $2 \sigma$ exclusion limit considering $300 \mathrm{fb}^{-1}$ integrated luminosity. In the same figure, current limit on top squark search through dilepton channel is shown in

\footnotetext{
${ }^{1}$ We already clarified earlier that the extreme small choice of any of these $\Delta M_{i j}$ can push this lower line anywhere below by making the analyzed channel undetectable in present search. Hence this band represents between most optimistic scenario to the extend this signature is promising at the LHC [73].
} 


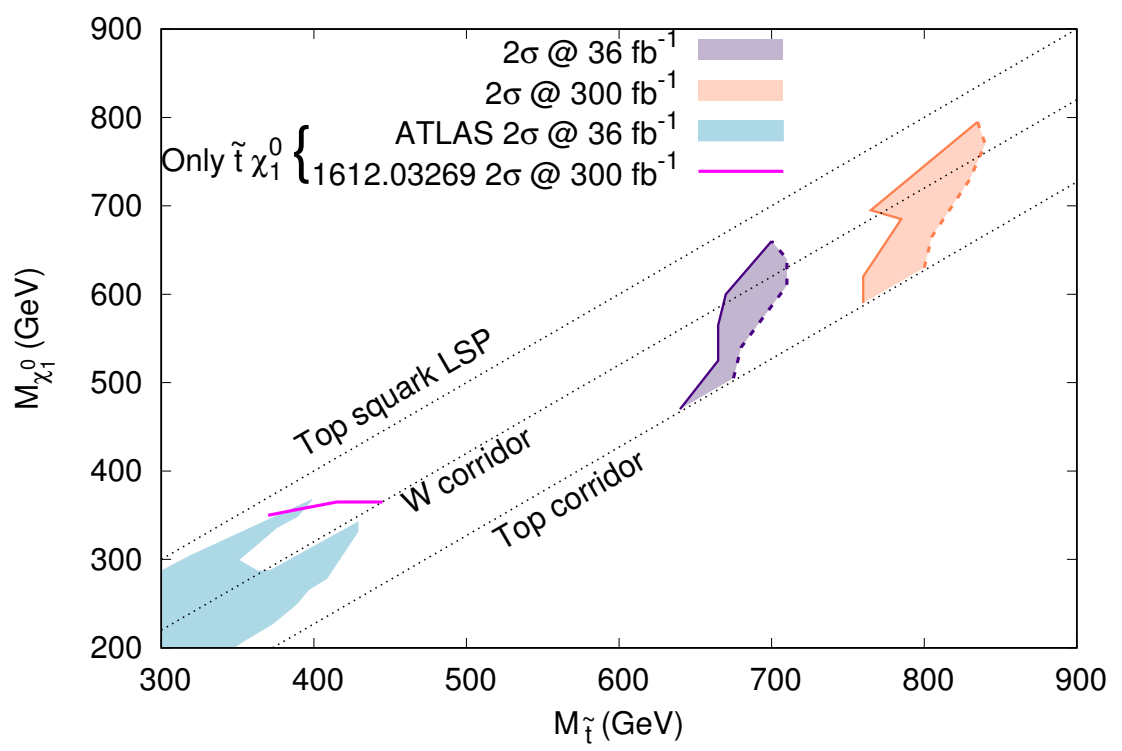

Figure 5. $95 \%$ exclusion limit in $M_{\tilde{t}}-M_{\chi_{1}^{0}}$ plane considering integrated luminosity as $36 \mathrm{fb}^{-1}$ and $300 \mathrm{fb}^{-1}$ with light purple shade and light orange shade respectively. Limits are obtained from tri-lepton $+\mathbb{E}_{T}$ final state originated from $\tilde{t}$ decay with $\chi_{1}^{ \pm}, \chi_{2}^{0}$ as intermediate states.

light blue patch as reported by the ATLAS collaboration [52]. In our previous work [56] we have studied the utility of the kinematic variables $R_{\ell E}$ and $R_{b E}$ for the compressed scenario where the top squark undergoes a four-body final state decay. The limit on top squark mass with data equivalent to integrated luminosity of $300 \mathrm{fb}^{-1}$ is shown in magenta curve. However, these two search limits assume that top squark to be the NLSP in contrary to the spectrum we are concerned here: $M_{\tilde{t}}>M_{\chi_{1}^{+}}>M_{\tilde{\ell} / \tilde{\nu}}>M_{\chi_{1}^{0}}$ and these limits are shown for reference only. Effective kinematic variables and absence of leptonic $W$ boson decay in the signal process help to achieve a higher limit in the present search channel.

Another important aspect of the present SUSY spectrum is that it is capable of providing strong limit on electroweakinos owing to strong production of top squarks which decay solely to charginos. In figure 6 we depict the exclusion limit in chargino-neutralino plane at $13 \mathrm{TeV}$ LHC with different integrated luminosities. We present the exclusion limits with 95\% CLs corresponding to $36 \mathrm{fb}^{-1}$ luminosity by the purple line, whereas the limit corresponding to $300 \mathrm{fb}^{-1}$ luminosity is shown by the green line. The red line depicts the current CMS search limit $[74,75]$ at $36 \mathrm{fb}^{-1}$ which is obtained from tri-lepton $+\mathbb{E}_{T}$ final state originated from the decay of $\chi_{1}^{ \pm} \chi_{2}^{0}$. However, one should keep in mind that in the CMS study the electroweakinos are produced via weak interaction. There is no experimental search in the dileptonic decay of stop squark where both chargino and sleptons are lighter than the top squark. However, in [74] with $36 \mathrm{fb}^{-1}$ luminosity data the CMS collaboration put limit on chargino mass in the compressed region, represented by magenta line, where the chargino is produced in the strong production via top squark and decays to $W$ boson and the lightest neutralino. Evidently, our results compliment the current experimental exclusion limits in the regions where there are no existing bounds (very weak bounds). Note that the CMS line shown in figure 6 is also after considering the similar choice of 


\begin{tabular}{|c|c|c|c|c|c|c|c|}
\hline \multicolumn{2}{|c|}{ Parameters } & \multicolumn{3}{|c|}{ Kinematic Variables } & \multicolumn{3}{|c|}{ Limit on top squark mass $(\mathrm{GeV})$} \\
\hline $\begin{array}{r}\Delta M_{\tilde{t} \chi_{1}^{+}} \\
(\mathrm{GeV})\end{array}$ & $\begin{array}{c}\Delta M_{\chi_{1}^{+} \tilde{\ell}} \\
(\mathrm{GeV})\end{array}$ & $\begin{array}{l}M_{b \ell}^{\max } \\
(\mathrm{GeV})\end{array}$ & $R_{\ell E}^{\max }$ & $R_{b E}^{\max }$ & $2 \sigma @ 36 \mathrm{fb}^{-1}$ & $2 \sigma @ 300 \mathrm{fb}^{-1}$ & $5 \sigma @ 300 \mathrm{fb}^{-1}$ \\
\hline \multirow{6}{*}{20} & 10 & 28 & 0.105 & \multirow{6}{*}{0.2} & 700 & 835 & 715 \\
\hline & 15 & 35 & 0.126 & & 680 & 815 & 700 \\
\hline & 25 & 45 & 0.175 & & 670 & 765 & 685 \\
\hline & 40 & 58 & 0.246 & & 665 & 785 & 680 \\
\hline & 60 & 72 & 0.339 & & 665 & 790 & 680 \\
\hline & 75 & 81 & 0.410 & & 640 & 760 & 655 \\
\hline \multirow{4}{*}{30} & 20 & 50 & 0.152 & \multirow{4}{*}{0.2} & 710 & 840 & 725 \\
\hline & 35 & 67 & 0.222 & & 705 & 830 & 720 \\
\hline & 55 & 84 & 0.316 & & 680 & 805 & 695 \\
\hline & 70 & 96 & 0.387 & & 675 & 795 & 690 \\
\hline \multirow{4}{*}{40} & 15 & 50 & 0.125 & \multirow{4}{*}{0.2} & 700 & 825 & 715 \\
\hline & 30 & 71 & 0.199 & & 700 & 830 & 720 \\
\hline & 50 & 93 & 0.293 & & 675 & 800 & 695 \\
\hline & 65 & 107 & 0.363 & & 675 & 795 & 685 \\
\hline \multirow{5}{*}{50} & 10 & 46 & 0.105 & \multirow{5}{*}{0.2} & 680 & 805 & 695 \\
\hline & 25 & 73 & 0.175 & & 710 & 840 & 725 \\
\hline & 35 & 85 & 0.222 & & 690 & 820 & 705 \\
\hline & 45 & 99 & 0.269 & & 670 & 760 & 685 \\
\hline & 60 & 116 & 0.339 & & 675 & 800 & 695 \\
\hline \multirow{4}{*}{60} & 20 & 72 & 0.152 & \multirow{4}{*}{0.2} & 680 & 805 & 695 \\
\hline & 40 & 103 & 0.246 & & 665 & 790 & 680 \\
\hline & 45 & 105 & 0.269 & & 670 & 790 & 685 \\
\hline & 55 & 122 & 0.316 & & 665 & 785 & 680 \\
\hline \multirow{3}{*}{70} & 15 & 67 & 0.128 & \multirow{3}{*}{0.2} & 685 & 810 & 700 \\
\hline & 35 & 105 & 0.222 & & 665 & 790 & 680 \\
\hline & 50 & 126 & 0.293 & & 650 & 765 & 665 \\
\hline
\end{tabular}

Table 2. Different benchmark points analyzed in the present study characterized by two mass gaps $\Delta M_{\tilde{t} \chi_{1}^{+}}$and $\Delta M_{\chi_{1}^{+} \tilde{\ell}}$ satisfying a compressed mass gap between LCP and LSP as, $\Delta M_{\tilde{t} \chi_{1}^{0}}=\Delta M_{\tilde{t} \chi_{1}^{+}}+$ $2 \times \Delta M_{\chi_{1}^{+} \tilde{\ell}}$. According to the mass differences various cuts are fixed (see, equations (2.6), (2.7) and (3.4) with following text) and are tabulated in the kinematic variables column. Using these cuts we have calculated the top squark mass reach at $13 \mathrm{TeV}$ LHC with integrated luminosity of $36 \mathrm{fb}^{-1}$ and $300 \mathrm{fb}^{-1}$. 


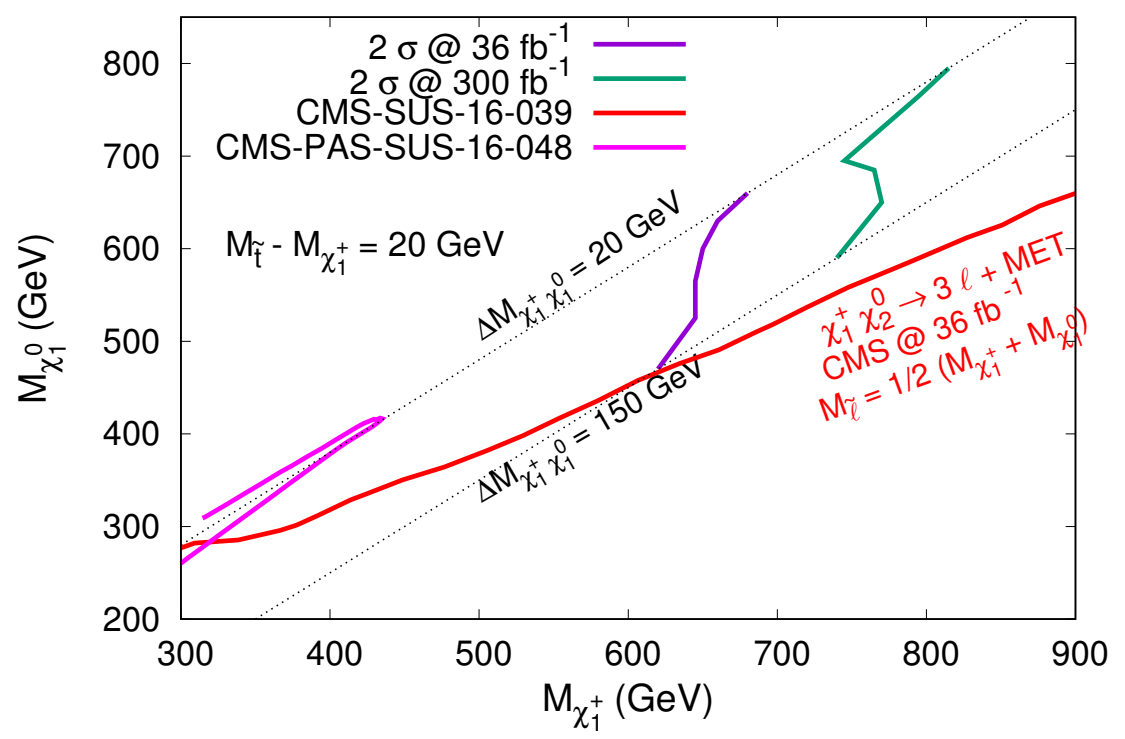

Figure 6. Exclusion limit in chargino-neutralino plane at $13 \mathrm{TeV}$ LHC considering compressed spectrum with top squark as lightest color sparticle. In order to show the efficacy of our new observables we have presented the exclusion limits for chargino with $95 \%$ CLs corresponding to $36 \mathrm{fb}^{-1}$ luminosity as displayed by the purple line. The limits corresponding to $300 \mathrm{fb}^{-1}$ luminosity is shown by the green line. The red line depicts the current CMS search limit where they have studied the trilepton along with missing energy considering direct production of chargino-neutralino. There is no experimental search in the dileptonic decay of stop squark where both chargino and sleptons are lighter than the top squark. However, in [74] with $36 \mathrm{fb}^{-1}$ luminosity data the CMS collaboration put limit on chargino mass in the compressed region where the chargino is produced in the strong production via top squark and decays to a $W$ boson and the lightest neutralino. The limit is displayed in magenta line.

mass gap $M_{\tilde{\ell}}=\alpha M_{\chi_{1}^{+}}+(1-\alpha) M_{\chi_{1}^{0}}$ with $\alpha=0.5$ as we have pointed in our benchmark. In the same reference [75] it is also demonstrated with other extreme mass gap possibilities like $\alpha=0.95,0.05$ make the reach extremely poor due to soft leptons. In this study we can exclude the chargino mass up to $680 \mathrm{GeV}$ for neutralino mass of $660 \mathrm{GeV}$ for $13 \mathrm{TeV}$ with $36 \mathrm{fb}^{-1}$ of integrated luminosity. Similarly, as evident from our model assumptions, the slepton mass up to $670 \mathrm{GeV}$ can also be excluded for the same data.

Finally, we have tabulated all the benchmark points we have analyzed in table 2. As we have already stated we vary the mass difference $\Delta M_{\tilde{t}_{\chi_{1}^{+}}}$and $\Delta M_{\chi_{1}^{+} \tilde{\ell}}$ in a controlled way to scan the whole parameter space. For all the benchmark points we have estimated LHC exclusion limit at $13 \mathrm{TeV}$ with integrated luminosity of $36 \mathrm{fb}^{-1}$ and $300 \mathrm{fb}^{-1}$. Also we have analyzed the discovery limit with integrated luminosity of $300 \mathrm{fb}^{-1}$.

In passing by, we would like to mention that our variables can be useful in dedicated electroweakinos searches [74] as well. Since signal and background topology for those searches are different, so one to one correspondence is not possible. However, keeping the compressed kinematics with equal velocity approximation in mind, we can still emphasize that part of the observable discussed in our work, if not all, will be useful to disentangle the background and signal events efficiently for those searches. 


\section{Summary and conclusion}

Presence of a light top squark $\left(M_{\tilde{t}}<1 \mathrm{TeV}\right)$ is crucial for the naturalness of the Standard Model. Search for such a top squark at the LHC is very important and several studies are done by the CMS and ATLAS collaboration to put limit on the mass of the top squark which is very close to TeV scale. However, if the SUSY spectrum is compressed, the limit can go down significantly. Several experimental as well as theoretical efforts poured into searching such compressed top squark recently. Most of the studies for compressed top squark assume a simplified spectrum where top squark is the NLSP and the lightest neutralino being the LSP. There can be other possibilities where one or more intermediate states can be present in between such top squark and LSP. This may change the search strategies at the LHC and all these spectra need to be explored to carry out an exhaustive search for the top squark.

One such possibility is having chargino and slepton in between top squark and the LSP while keeping the mass spectrum compressed. In this case, top squark decay dominantly to a dileptonic final state along with missing energy from invisible LSPs and the SM neutrinos. Since the spectrum is compressed, all the final state particles are soft and it is very difficult to detect them. On the other hand the dominant background is $t \bar{t}$ which is produced abundantly at the LHC owing to its huge production cross-section. Hence it is very important to minimize the background events efficiently in order to achieve a stringent limit on the top squark mass in the dileptonic scenario. Kinematics of the signal and the background processes can be used to separate them and in this paper we have studied potency of several kinematic variables which can be used to minimize the background. We notice that these variables encode the information about mass gaps between the top squark-chargino and slepton-neutralino pair and they are capable of providing a stringent limit on the top squark mass. We observe that depending on the mass gaps $\Delta M_{\tilde{t} \chi_{1}^{+}}$and $\Delta M_{\chi_{1}^{+} \tilde{\ell}}$ a top squark with mass from $640 \mathrm{GeV}$ to $715 \mathrm{GeV}$ can be excluded at $2 \sigma$ with an integrated luminosity of $36 \mathrm{fb}^{-1}$ data at the $13 \mathrm{TeV}$ LHC. Similarly, the exclusion limit for the chargino achieved from this analysis is 680 for neutralino mass of $660 \mathrm{GeV}$ and comparable limit for slepton (sneutrino) can also be obtained. The exclusion limit goes up to $840 \mathrm{GeV}$ for $300 \mathrm{fb}^{-1}$ data and at that luminosity it is possible to discover a top squark at $5 \sigma$ with mass up to $725 \mathrm{GeV}$.

\section{Acknowledgments}

This work was partially supported by funding available from the Department of Atomic Energy, Government of India, for the Regional Centre for Accelerator-based Particle Physics (RECAPP), Harish-Chandra Research Institute; TDP project at Physical Research Laboratory, Department of Space, Government of India. AKS acknowledges the support from Department of Science and Technology, Government of India under the fellowship reference number PDF/2017/002935 (SERB National Post Doctoral Fellowship). AKS also thank the Indian Association for the Cultivation of Science (IACS), Kolkata for administrative support. 


\section{A Benchmark points}

In this appendix, we display two benchmark points with low scale input parameters and the relevant sparticle masses for the simplified model in our study. In both of these cases the neutralino is bino type and the lightest chargino is wino type. As shown in the table, the correct relic density can be obtained using A-funnel region where $m_{A} \sim 2 m_{\chi_{1}^{0}}$. With a tuned $\tan \beta$ and trilinear coupling Higgs mass constraint is satisfied. We have fixed the gaugino mass parameter $M_{1}$ and varied $M_{2}$ and other soft SUSY breaking masses to achieve the desired mass spectrum as shown in the table. We have also satisfied the low energy flavor physics constraints, $b \rightarrow s \gamma$ and $B_{s} \rightarrow \mu \mu$ within $2 \sigma$ limit.

\begin{tabular}{|c|c|c|}
\hline Parameters & BP1 & BP2 \\
\hline$\Delta M_{\tilde{t}_{1}^{+}} ; \Delta M_{\chi_{1}^{+} \tilde{\ell}} \approx \Delta M_{\tilde{\ell}_{1}^{0}}$ & $30 ; 18$ & $50 ; 45$ \\
\hline$M_{1} ; M_{2}$ & $500 ; 515$ & $500 ; 568$ \\
$M_{3} ; A_{t} ; \mu ; \tan \beta$ & $3000 ;-3000 ;-2000 ; 20$ \\
\hline$m_{\tilde{g}} ; m_{\widetilde{q}_{L}} ; m_{\widetilde{q}_{R}}$ & $2882 ; 2166 ; 2162$ \\
$m_{\widetilde{t}_{2}} ; m_{\widetilde{b}_{1}} ; m_{\widetilde{b}_{2}}$ & $2159 ; 2129 ; 2181$ \\
$m_{\widetilde{\ell}_{R}} ; m_{\widetilde{\tau}_{1}} ; m_{\widetilde{\tau}_{2}}$ & $2205 ; 2188 ; 2197$ \\
\hline$m_{\widetilde{t}_{1}}$ & 570.3 & 645.2 \\
$m_{\widetilde{\chi}_{1}^{ \pm}}$ & 541.5 & 595.6 \\
$m_{\widetilde{\ell}_{L}} ; m_{\widetilde{\nu}_{L}}$ & $519.3 ; 513.3$ & $548.0 ; 542.1$ \\
$m_{\widetilde{\chi}_{1}^{0}}$ & 501.9 & 502.0 \\
\hline$m_{h}$ & 123.9 & 123.9 \\
$m_{A}$ & 1015.0 & 1013.0 \\
$\Omega^{2}$ & 0.1102 & 0.1144 \\
\hline $\operatorname{BR}(b \rightarrow s \gamma)$ & \multicolumn{2}{|c|}{$3.57 \times 10^{-4}$} \\
$\operatorname{BR}\left(B_{s} \rightarrow \mu \mu\right)$ & \multicolumn{2}{|c|}{$2.77 \times 10^{-9}$} \\
\hline
\end{tabular}

Table 3. Low scale input parameters and the relevant sparticle masses for two of the chosen benchmark points satisfying all the collider, DM and low energy constraints. All the masses and dimensionful parameters are written in $\mathrm{GeV}$ units. 
Open Access. This article is distributed under the terms of the Creative Commons Attribution License (CC-BY 4.0), which permits any use, distribution and reproduction in any medium, provided the original author(s) and source are credited.

\section{References}

[1] CMS collaboration, Observation of a new boson at a mass of $125 \mathrm{GeV}$ with the CMS experiment at the LHC, Phys. Lett. B 716 (2012) 30 [arXiv:1207.7235] [INSPIRE].

[2] ATLAS collaboration, Observation of a new particle in the search for the Standard Model Higgs boson with the ATLAS detector at the LHC, Phys. Lett. B 716 (2012) 1 [arXiv: 1207.7214] [INSPIRE].

[3] ATLAS collaboration, Search for a Scalar Partner of the Top Quark in the Jets+ETmiss Final State at $\sqrt{s}=13 \mathrm{TeV}$ with the ATLAS detector, ATLAS-CONF-2017-020 (2017).

[4] CMS collaboration, Search for supersymmetry using hadronic top quark tagging in 13 TeV pp collisions, CMS-PAS-SUS-16-050 (2017).

[5] J. Dutta, P. Konar, S. Mondal, B. Mukhopadhyaya and S.K. Rai, A Revisit to a Compressed Supersymmetric Spectrum with 125 GeV Higgs, JHEP 01 (2016) 051 [arXiv:1511.09284] [INSPIRE].

[6] J. Dutta, P. Konar, S. Mondal, B. Mukhopadhyaya and S.K. Rai, Search for a compressed supersymmetric spectrum with a light Gravitino, JHEP 09 (2017) 026 [arXiv:1704.04617] [INSPIRE].

[7] CMS collaboration, Search for direct top squark pair production in the all-hadronic final state in proton-proton collisions at $\sqrt{s}=13 \mathrm{TeV}$, CMS-PAS-SUS-16-049 (2017).

[8] C.G. Lester and D.J. Summers, Measuring masses of semiinvisibly decaying particles pair produced at hadron colliders, Phys. Lett. B 463 (1999) 99 [hep-ph/9906349] [INSPIRE].

[9] W.S. Cho, K. Choi, Y.G. Kim and C.B. Park, Gluino Stransverse Mass, Phys. Rev. Lett. 100 (2008) 171801 [arXiv:0709.0288] [INSPIRE].

[10] Y. Bai, H.-C. Cheng, J. Gallicchio and J. Gu, Stop the Top Background of the Stop Search, JHEP 07 (2012) 110 [arXiv:1203.4813] [INSPIRE].

[11] C. Kilic and B. Tweedie, Cornering Light Stops with Dileptonic mT2, JHEP 04 (2013) 110 [arXiv: 1211.6106] [INSPIRE].

[12] J. Cao, C. Han, L. Wu, J.M. Yang and Y. Zhang, Probing Natural SUSY from Stop Pair Production at the LHC, JHEP 11 (2012) 039 [arXiv:1206.3865] [INSPIRE].

[13] P. Konar, K. Kong and K.T. Matchev, $\sqrt{\hat{s}}_{\text {min }}$ : A global inclusive variable for determining the mass scale of new physics in events with missing energy at hadron colliders, JHEP 03 (2009) 085 [arXiv: 0812.1042] [INSPIRE].

[14] P. Konar, K. Kong, K.T. Matchev and M. Park, RECO level $\sqrt{s}_{\text {min }}$ and subsystem $\sqrt{s}_{\text {min }}$ : Improved global inclusive variables for measuring the new physics mass scale in $\mathbb{E}_{T}$ events at hadron colliders, JHEP 06 (2011) 041 [arXiv:1006. 0653] [INSPIRE].

[15] ATLAS collaboration, Search for light top squark pair production in final states with leptons and $b^{-}$jets with the ATLAS detector in $\sqrt{s}=7 \mathrm{TeV}$ proton-proton collisions, Phys. Lett. B 720 (2013) 13 [arXiv:1209.2102] [INSPIRE]. 
[16] CMS collaboration, Search for gluino mediated bottom-and top-squark production in multijet final states in pp collisions at 8 TeV, Phys. Lett. B 725 (2013) 243 [arXiv:1305.2390] [INSPIRE].

[17] I. Hinchliffe, F.E. Paige, M.D. Shapiro, J. Soderqvist and W. Yao, Precision SUSY measurements at CERN LHC, Phys. Rev. D 55 (1997) 5520 [hep-ph/9610544] [INSPIRE].

[18] B.K. Gjelsten, D.J. Miller and P. Osland, Measurement of SUSY masses via cascade decays for SPS 1a, JHEP 12 (2004) 003 [hep-ph/0410303] [INSPIRE].

[19] C. Rogan, Kinematical variables towards new dynamics at the LHC, arXiv:1006.2727 [INSPIRE].

[20] CMS collaboration, Inclusive search for supersymmetry using the razor variables in $p p$ collisions at $\sqrt{s}=7$ TeV, Phys. Rev. Lett. 111 (2013) 081802 [arXiv:1212.6961] [INSPIRE].

[21] CMS collaboration, Search for Supersymmetry Using Razor Variables in Events with b-Tagged Jets in pp Collisions at $\sqrt{s}=8$ TeV, Phys. Rev. D 91 (2015) 052018 [arXiv: 1502.00300] [INSPIRE].

[22] L. Randall and D. Tucker-Smith, Dijet Searches for Supersymmetry at the LHC, Phys. Rev. Lett. 101 (2008) 221803 [arXiv:0806.1049] [INSPIRE].

[23] CMS collaboration, Search for top squark pair production in compressed-mass-spectrum scenarios in proton-proton collisions at $\sqrt{s}=8 \mathrm{TeV}$ using the $\alpha_{T}$ variable, Phys. Lett. B 767 (2017) 403 [arXiv: 1605.08993] [INSPIRE].

[24] M. Drees, M. Hanussek and J.S. Kim, Light Stop Searches at the LHC with Monojet Events, Phys. Rev. D 86 (2012) 035024 [arXiv: 1201.5714] [InSPIRE].

[25] M. Carena, A. Freitas and C.E.M. Wagner, Light Stop Searches at the LHC in Events with One Hard Photon or Jet and Missing Energy, JHEP 10 (2008) 109 [arXiv:0808.2298] [INSPIRE].

[26] S. Bornhauser, M. Drees, S. Grab and J.S. Kim, Light Stop Searches at the LHC in Events with two b-Jets and Missing Energy, Phys. Rev. D 83 (2011) 035008 [arXiv:1011.5508] [INSPIRE].

[27] A. Delgado, G.F. Giudice, G. Isidori, M. Pierini and A. Strumia, The light stop window, Eur. Phys. J. C 73 (2013) 2370 [arXiv:1212.6847] [InSPIRE].

[28] K. Krizka, A. Kumar and D.E. Morrissey, Very Light Scalar Top Quarks at the LHC, Phys. Rev. D 87 (2013) 095016 [arXiv:1212.4856] [INSPIRE].

[29] G. Ferretti, R. Franceschini, C. Petersson and R. Torre, Spot the stop with a b-tag, Phys. Rev. Lett. 114 (2015) 201801 [arXiv:1502.01721] [INSPIRE].

[30] M. Drees and M.M. Nojiri, A new signal for scalar top bound state production, Phys. Rev. Lett. 72 (1994) 2324 [hep-ph/9310209] [INSPIRE].

[31] S.P. Martin, Diphoton decays of stoponium at the Large Hadron Collider, Phys. Rev. D 77 (2008) 075002 [arXiv:0801.0237] [INSPIRE].

[32] G.H. Duan, L. Wu and R. Zheng, Resonant Higgs pair production as a probe of stop at the LHC, JHEP 09 (2017) 037 [arXiv:1706.07562] [InSPIRE].

[33] M. Perelstein and C. Spethmann, A collider signature of the supersymmetric golden region, JHEP 04 (2007) 070 [hep-ph/0702038] [INSPIRE].

[34] D. Ghosh, Boosted dibosons from mixed heavy top squarks, Phys. Rev. D 88 (2013) 115013 [arXiv: 1308.0320] [INSPIRE]. 
[35] CMS collaboration, Search for top-squark pairs decaying into Higgs or $Z$ bosons in pp collisions at $\sqrt{s}=8 \mathrm{TeV}$, Phys. Lett. B 736 (2014) 371 [arXiv:1405.3886] [InSPIRE].

[36] Z. Kang, J. Li and M. Zhang, Uncover Compressed Supersymmetry via Boosted Bosons from the Heavier Stop/Sbottom, Eur. Phys. J. C 77 (2017) 371 [arXiv:1703.08911] [InSPIRE].

[37] B.P. Padley, K. Sinha and K. Wang, Natural Supersymmetry, Muon $g-2$ and the Last Crevices for the Top Squark, Phys. Rev. D 92 (2015) 055025 [arXiv:1505.05877] [InSPIRE].

[38] H.-C. Cheng, C. Gao and L. Li, Compressed Stop Searches with Two Leptons and Two b-jets, arXiv:1706.02805 [INSPIRE].

[39] K.-i. Hikasa and M. Kobayashi, Light Scalar Top at $e^{+} e^{-}$Colliders, Phys. Rev. D 36 (1987) 724 [INSPIRE].

[40] C. Boehm, A. Djouadi and Y. Mambrini, Decays of the lightest top squark, Phys. Rev. D 61 (2000) 095006 [hep-ph/9907428] [INSPIRE].

[41] M. Muhlleitner and E. Popenda, Light Stop Decay in the MSSM with Minimal Flavour Violation, JHEP 04 (2011) 095 [arXiv:1102.5712] [INSPIRE].

[42] CMS collaboration, Searches for third-generation squark production in fully hadronic final states in proton-proton collisions at $\sqrt{s}=8 \mathrm{TeV}, \mathrm{JHEP} 06$ (2015) 116 [arXiv:1503.08037] [INSPIRE].

[43] CMS collaboration, A search for new phenomena in pp collisions at $\sqrt{s}=13 \mathrm{TeV}$ in final states with missing transverse momentum and at least one jet using the $\alpha_{\mathrm{T}}$ variable, Eur. Phys. J. C 77 (2017) 294 [arXiv: 1611.00338] [INSPIRE].

[44] ATLAS collaboration, Search for pair-produced third-generation squarks decaying via charm quarks or in compressed supersymmetric scenarios in pp collisions at $\sqrt{s}=8 \mathrm{TeV}$ with the ATLAS detector, Phys. Rev. D 90 (2014) 052008 [arXiv:1407.0608] [INSPIRE].

[45] ATLAS collaboration, ATLAS Run 1 searches for direct pair production of third-generation squarks at the Large Hadron Collider, Eur. Phys. J. C 75 (2015) 510 [Erratum ibid. C 76 (2016) 153] [arXiv:1506.08616] [INSPIRE].

[46] CMS collaboration, Search for direct production of supersymmetric partners of the top quark in the all-jets final state in proton-proton collisions at $\sqrt{s}=13 \mathrm{TeV}$, JHEP 10 (2017) 005 [arXiv: 1707.03316] [INSPIRE].

[47] CMS collaboration, Search for supersymmetry in events with soft leptons, low jet multiplicity and missing transverse energy in proton-proton collisions at $\sqrt{s}=8 \mathrm{TeV}$, Phys. Lett. B 759 (2016) 9 [arXiv: 1512.08002] [INSPIRE].

[48] ATLAS collaboration, Search for top squark pair production in final states with one isolated lepton, jets and missing transverse momentum in $\sqrt{s}=8 \mathrm{TeV}$ pp collisions with the ATLAS detector, JHEP 11 (2014) 118 [arXiv:1407.0583] [INSPIRE].

[49] CMS collaboration, Search for supersymmetry in events with at least one soft lepton, low jet multiplicity and missing transverse momentum in proton-proton collisions at $\sqrt{s}=13 \mathrm{TeV}$, CMS-PAS-SUS-16-031 (2016).

[50] CMS collaboration, Search for new physics in the compressed mass spectra scenario using events with two soft opposite-sign leptons and missing transverse momentum at $\sqrt{s}=13 \mathrm{TeV}$, CMS-PAS-SUS-16-025 (2016).

[51] CMS collaboration, Search for supersymmetry in events with at least one soft lepton, low jet multiplicity and missing transverse momentum in proton-proton collisions at $\sqrt{s}=13 \mathrm{TeV}$, CMS-PAS-SUS-16-052 (2017). 
[52] ATLAS collaboration, Search for direct top squark pair production in final states with two leptons in $\sqrt{s}=13 \mathrm{TeV}$ pp collisions with the ATLAS detector, ATLAS-CONF-2017-034 (2017).

[53] H. An and L.-T. Wang, Opening up the compressed region of top squark searches at $13 \mathrm{TeV}$ LHC, Phys. Rev. Lett. 115 (2015) 181602 [arXiv:1506. 00653] [InSPIRE].

[54] K. Hagiwara and T. Yamada, Equal-velocity scenario for hiding dark matter at the LHC, Phys. Rev. D 91 (2015) 094007 [arXiv:1307.1553] [INSPIRE].

[55] H.-C. Cheng, C. Gao, L. Li and N.A. Neill, Stop Search in the Compressed Region via Semileptonic Decays, JHEP 05 (2016) 036 [arXiv:1604.00007] [INSPIRE].

[56] P. Konar, T. Mondal and A.K. Swain, Demystifying the compressed top squark region with kinematic variables, Phys. Rev. D 96 (2017) 095011 [arXiv:1612.03269] [INSPIRE].

[57] D.J. Miller, P. Osland and A.R. Raklev, Invariant mass distributions in cascade decays, JHEP 03 (2006) 034 [hep-ph/0510356] [INSPIRE].

[58] J. Alwall et al., The automated computation of tree-level and next-to-leading order differential cross sections and their matching to parton shower simulations, JHEP 07 (2014) 079 [arXiv: 1405.0301] [INSPIRE].

[59] NNPDF collaboration, R.D. Ball et al., Parton distributions for the LHC Run II, JHEP 04 (2015) 040 [arXiv:1410.8849] [INSPIRE].

[60] http://cp3.irmp.ucl.ac.be/projects/madgraph/wiki/FAQ-General-13.

[61] T. Sjöstrand, S. Mrenna and P.Z. Skands, PYTHIA 6.4 Physics and Manual, JHEP 05 (2006) 026 [hep-ph/0603175] [INSPIRE].

[62] T. Sjöstrand, S. Mrenna and P.Z. Skands, A Brief Introduction to PYTHIA 8.1, Comput. Phys. Commun. 178 (2008) 852 [arXiv:0710.3820] [INSPIRE].

[63] DELPHES 3 collaboration, J. de Favereau et al., DELPHES 3, A modular framework for fast simulation of a generic collider experiment, JHEP 02 (2014) 057 [arXiv:1307.6346] [INSPIRE].

[64] M.L. Mangano, M. Moretti, F. Piccinini and M. Treccani, Matching matrix elements and shower evolution for top-quark production in hadronic collisions, JHEP 01 (2007) 013 [hep-ph/0611129] [INSPIRE].

[65] S. Hoeche et al., Matching parton showers and matrix elements, in HERA and the LHC: A workshop on the implications of HERA for LHC physics: Proceedings Part A, pp. 288-289 (2005) [CERN-2005-014.288] [hep-ph/0602031] [INSPIRE].

[66] S. Padhi, LHC SUSY Cross Section Working Group, https://twiki.cern.ch/twiki/bin/view/LHCPhysics/SUSYCrossSections.

[67] C. Borschensky et al., Squark and gluino production cross sections in pp collisions at $\sqrt{s}=13,14,33$ and 100 TeV, Eur. Phys. J. C 74 (2014) 3174 [arXiv:1407.5066] [InSPIRE].

[68] M. Czakon and A. Mitov, Top++: A Program for the Calculation of the Top-Pair Cross-Section at Hadron Colliders, Comput. Phys. Commun. 185 (2014) 2930 [arXiv:1112.5675] [INSPIRE].

[69] N. Kidonakis, Theoretical results for electroweak-boson and single-top production, PoS (DIS2015) 170 [arXiv: 1506. 04072] [INSPIRE].

[70] M. Cacciari, G.P. Salam and G. Soyez, The anti- $k_{t}$ jet clustering algorithm, JHEP 04 (2008) 063 [arXiv: 0802.1189] [INSPIRE]. 
[71] CMS collaboration, Identification of b-quark jets with the CMS experiment, 2013 JINST 8 P04013 [arXiv: 1211.4462] [INSPIRE].

[72] CMS SUSY results: Objects efficiency, https://twiki.cern.ch/twiki/bin/view/CMSPublic/SUSICHEP2016ObjectsEfficiency.

[73] P. Konar, K.T. Matchev, M. Park and G.K. Sarangi, How to look for supersymmetry under the lamppost at the LHC, Phys. Rev. Lett. 105 (2010) 221801 [arXiv:1008.2483] [InSPIRE].

[74] CMS collaboration, Search for new physics in events with two soft oppositely charged leptons and missing transverse momentum in proton-proton collisions at $\sqrt{s}=13 \mathrm{TeV}$, CMS-PAS-SUS-16-048 [arXiv: 1801.01846] [INSPIRE].

[75] CMS Collaboration, Search for electroweak production of charginos and neutralinos in multilepton final states in proton-proton collisions at $\sqrt{s}=13$ TeV, CMS-SUS-16-039 (2017). 